\title{
The term (كلمة) in the Holy Quran \\ Between Translation and interpretation
}

\author{
Jamal Abdulhakeem Abdullah \\ Instructor \\ Mustansiryah University \\ College of Arts \\ Département of French Language \\ ij200064@gmail.com \\ 07702623722
}

\section{DOI: $\underline{10.31973 / \text { aj.v1i136.1103 }}$}

\begin{abstract}
There is no doubt that the correct and accurate translation of religious terms and concepts is of the utmost importance for those who seek to be precise in their translations and strive to convey the meanings to the readers of the language transmitted with extreme precision. and linguistic proficiency, taking into account the linguistic and cultural stock of the transmitted language, as this is a major condition for its successful Translation.

This research seeks to highlight the rhetoric of the Koran by touching on the polysemy of a term in the translation. In this research, the term "word" is treated because it is mentioned in 22 verses in different surahs of the Quran.

After clarifying what translation and interpretation are, we give an overview of the history of Quran translation and mention the first to translate the Quran into French and identify the three corpuses on which the research depends (translations of the Quran in French).

In order to obtain the exact result of these translations, two versions of the interpretations of these verses are consulted. To clearly show the results of this research are represented in tabular and graphical form.

The conclusion of the research which was reached is that the use by the translator of the Quran interpretation books of the Quranic verses is very necessary in order to follow the rhetoric of the Quran and get an accurate translation.

In conclusion, it is hoped that this simple research will help support research efforts on translation in general and Quran translation in particular.
\end{abstract}

Keywords: Eloquence - Interpretation -Polysemy - Term Translation 
Terme (كلمة) dans le Coran saint

Entre la traduction et l'interprétation

\section{Sommaire}

\section{Instructeur: Jamal Abdulhakeem Abdullah \\ Université Al-Mustansiryah \\ Faculté des lettres \\ Département de Français}

Il ne fait aucun doute que la traduction correcte et exacte des termes et concepts religieux est de la plus haute importance pour ceux qui cherchent à être précis dans leurs traductions et s'efforcent de transmettre les significations aux lecteurs de la langue transmise avec une extrême précision et une maîtrise linguistique, en tenant compte du stock linguistique et culturel de la langue transmise, car c'est une condition majeure de son succès Traduction.

Cette recherche cherche à mettre en évidence la rhétorique du Coran en touchant à la polysémie d'un terme dans la traduction. Dans cette recherche, le terme (《كلمة) «mot») est traité parce qu'il est mentionné dans 22 versets dans différentes sourates du Coran.

Après avoir clarifié ce que sont la traduction et l'interprétation, on donne un aperçu de l'histoire de la traduction du Coran et mentionné le premier à traduire le Coran en français et identifié les trois corpus dont la recherche dépend (traductions du Coran en français).

Afin d'obtenir le résultat exact de ces traductions, deux versions des interprétations de ces versets sont consultées. Pour bien montrer les résultats de cette recherche sont représentés en forme tabulaire et graphique.

La conclusion de la recherche qui a été atteinte est que l'utilisation par le traducteur des livres d'interprétation du Coran des versets coraniques est très nécessaire afin de suivre la rhétorique du Coran et d'obtenir une traduction exacte.

En conclusion, on espère que cette simple recherche contribuera à soutenir les efforts de recherche sur la traduction en général et la traduction du Coran en particulier.

Mots clés: Eloquence - Interprétation- polysémie- TermeTraduction

\section{- Introduction}

Il y a longtemps, les peuples et les nations ont compris la nécessité de se connecter les uns aux autres et à l'échange de la culture et de l'information. Sans la traduction, le monde devient des groupes humains dispersés. Par conséquent, la traduction est considérée comme moyen efficace d'apprendre et de voir la nature des 
civilisations, des sociétés et de différentes religions. La traduction des livres saints vise à atteindre ces objectifs.

Cette recherche vise à étudier la polysémie du terme arabe (كلمة) qui signifie littérairement de (mot). Alors, on provoque la question suivante : la traduction littérale ou celle qui dépend de l'interprétation répond aux exigences de l'éloquence du Coran ?

L'éloquence représente un aspect du miracle du Coran. Bucaille a montré dans son livre intitulé Torah, Bible, Coran et la science que :"Le Coran est un texte descendu de la révélation au prophète Muhammad par Seigneur des anges Jibril" (Bucaille, 1990, p. 18).

Evidemment, malgré leurs grandes éloquences, les arabes n'ont pas pu composer un livre comme le Coran: "Par le Coran, le Prophète (que la paix soit sur lui) a contesté les Arabes, peuples de l'éloquence, de composer comme le quel même une sourate mais ils ont échoué"(Arif, 1988, p. 115).

Ainsi, le Coran est le livre de Dieu dans sa descente, dans ses versets et dans l'opération de le garder :"Le mot Coran comprend deux sens :l'un est linguistique, c'est le nom verbal du verbe qara'a, dans le sens de lecture, tel qu'il est dit dans un des versets : "C'est à Nous qu'Incombe son assemblage et sa lecture. Quand Nous le Lisons, suis alors sa lecture » $(75: 17,18)$; 1'autre, est le nom propre même de ce Livre, Saint, Sacré et Immaculé : le Coran, dans le sens de la lecture par excellence. (Abdel Aziz Z. , 2009, p. 27)

Dans cette étude, on va éclaircir la nature de la traduction et l'interprétation puis on va donner un aperçu historique de la traduction du Coran. Ainsi, l'étude va se focaliser sur trois corpus de traduction pour étayer les résultats de cette recherche.

\section{- La traduction et l'interprétation}

La traduction aide l'humanité à communiquer et à bénéficier de leurs expériences. Le livre intitulé des papiers de recherche en traduction a d'un autre point de vue sur la traduction : "La traduction est une nécessité de la civilisation, une activité intellectuelle et un processus linguistique, imposée par le contact entre des peuples de langues différentes, soit un contact direct comme dans les guerres, dans les migrations et dans le colonialisme ou indirectement, comme celui qui se passe à travers les médias ou la communication" (AbdulLatif, 2014, p. 17) .

La précision de la traduction représente un problème pour ceux qui la pratiquent, donc il est nécessaire de transformer la traduction d'une opération linguistique simple à une procédure civilisée pratiquée consciemment et conscience préalables car pour traduire une idée de bon sens voulu de la langue source à la langue cible, on a besoin d'un grand effort . Le traducteur, c'est la personne qui modifie les pensées 
des autres dans une autre langue Anani dit:" Le traducteur est un auteur, son travail est la formulation d'idées en des mots adressées au lecteur, la différence entre l'auteur et lui est que les idées qu'il a écrit ne sont pas les siennes" (Anani, 1996, p. 5).

La traduction doit être le miroir clair pour refléter, complètement, le sens de toutes ses détails dans le texte original, en particulier, si le texte appartient au livre céleste ou religieux parce que celui-ci a une importance particulière. Dans ce cas, le traducteur doit chercher à s'intéresser à ce genre de traduction. Alors, la traduction du Coran exige un traitement spécial. D'ailleurs, l'interprétation, c'est la clarification des choses en modifiant leurs significations. Ce vocabulaire soulevé, dans cette recherche, signifie l'explication du Coran dans sa langue d'origine non dans une autre langue pour comprendre les versets du Coran, à savoir l'exégèse. A cet égard Abdul Azim a éclairci : "L'intéresse à l'explication continue jusqu'à ce jour [...] Y compris l'explication du Coran entier, l'explication d'une partie, d'une Sourate et d'un verset " (Al zarkany, 1995, p. 31)

En conséquence, la responsabilité du traducteur du Coran ne ressemble pas à celle de l'interprète; le traducteur n'a pas le droit d'éclaircir un mot vague, mais l'interprète possède ce droit.

\section{- Aperçu historique sur la traduction du Coran:}

La religion de l'islam encourage la traduction du Coran, même ce type de traduction a été considéré comme un devoir. La meilleure preuve c'est l'expression du prophète Mahomet dit: "Informez en mon nom, même si un verset" (Al-Othaimeen, 1429 Héjrie, p. 1426 )

Alors, sans la traduction, on ne peut pas informer et clarifier les éléments essentiels, les instructions corrects de l'islam et à éclaircir ses confusions aux autres nations étrangères.

Les opposants de traduire le Coran ont un autre point de vue; Ils posent certains arguments: ils disent que si nous permettons la traduction du Coran chaque nation aura, dans sa langue, une version privée, et elle sera bien différente que celles des autres nations. Par conséquent, la traduction du Coran au lieu d'aider à l'unité des musulmans, elle participera à la dispersion.

André Du Rayer, le consul français à Istanbul, est le premier traducteur du Coran en français. La version a été imprimée à Paris en 1647. Jalal Al-din a confirmé cette information: "La première traduction du Coran en français est une traduction de Sieur du Rayer imprimée en 1647 " (Al -Allush, 2008, p. 9). Cette version de traduction est imprimée plusieurs éditions jusqu'à l'année 1775, elle est devenue proche du texte original, elle a été considérée comme la source de traduction principale dans les langues européennes. Et on a 
publié de nombreuses traductions dans des langues différentes et sont réparties dans de nombreux pays.

\section{- Corpus}

Cette étude se fond sur 22 versets dans les quels trois traduction en français qui sont:

\section{1-Zeinab Abdel Aziz. Le Coran Traduction du sens de ses Versets}

Zainab Abdel Aziz professeur de civilisation française et de littérature française à l'Université du Caire, elle a travaillé comme assistante d'enseignement au Collège d'études islamiques d'Al-Azhar. Elle a fait la traduction du Coran en français plus récente que de la traduction de Jacques Berque; il s'agit de la première traduction publiée par un musulmane égyptienne. Cette traduction, par souci de brièveté, va symboliser par la lettre $(\mathbf{Z})$ dans cet article.

2 -Le NOBEL CORAN et la traduction en langue francaise de ses sens. Complexe Roi FAHAD pour l'impression du NOBEL CORAN :

Cette traduction est considérée comme l'une des meilleures traductions du Coran en .Elle a été publiée en 1420 AH par le Complexe du Roi Fahd pour l'impression du Noble Coran, à Médine. Il a été créé en $1403 \mathrm{AH}$ et a été inauguré en $1405 \mathrm{AH}$. Cette traduction va être $(\mathbf{F})$.

\section{3- Muhammad Hamidullah .Le Saint Coran:}

Muhammad Hamidullah né le 19 février 1908 - 17 décembre 2002), un spécialiste du droit islamique et un auteur universitaire avec plus de 250 livres. Il parlait couramment 22 langues dont l'ourdou (sa langue maternelle), persan, l'arabe, français, anglais, allemand, italien, grec, turc, russe, etc. Et il a appris le thaï à 84 ans. Sa traduction va symboliser par $(\mathbf{H})$.

- La fluctuation du terme (كلمة) :

\section{Première section: l'accord de la traduction}

Nous avons constaté que le terme كلمة a été traduit par le terme (parole, paroles) par les trois traductions dans les 11 versets suivants:

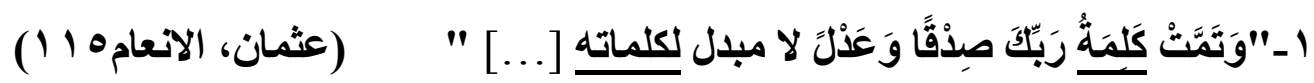
$\underline{\mathbf{Z}}$ :" La parole de ton Seigneur s'est accomplie véritablement et justement. Rien ne peut changer ses paroles [...]" (Abdel Aziz Z. , , 2009, p. 206).

F: " Et la parole de ton Seigneur s'est accomplie, en toute vérité et équité. Nul ne peut modifier ses paroles. [...]" (NOBEL, 1420 Héjrie, p. 142).

H:" Et la parole de ton Seigneur s'est accomplie en toute vérité et équité. Personne qui modifie ses paroles [...] (Hamidullah M. , , 1989, p. 142)." 


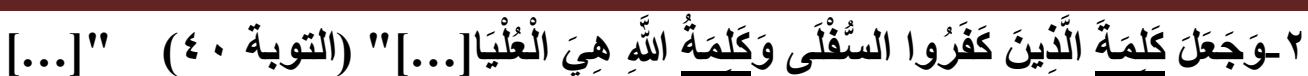

$\underline{\text { Z: }}$ " Fit la parole de ceux qui devinrent mécréants l'inférieure. La Parole d'Allah Est la Supérieure,." (AbdelAziz Z. , 2009, p. 259)

F: "Il baissa ainsi la parole des mécréants, tandis que la parole d'Allah eut le dessus. .." (LeNOBEL, 1420 Héjrie , p. 193)

H: "Fit plus basse la parole des mécréants, tandis que la parole de Dieu reste la plus haute." (Hamidullah, 1989, p. 194).

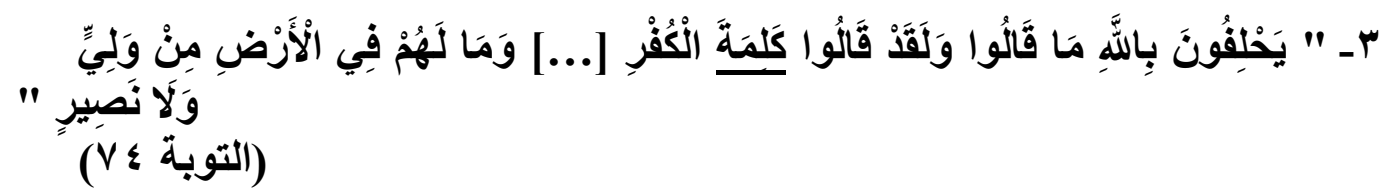

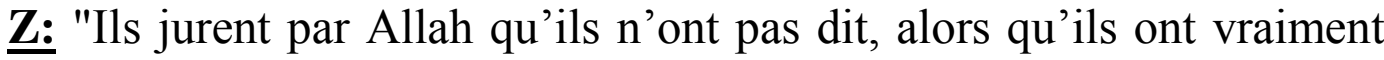
proféré la parole de mécréance [...] Et ils n'auront sur terre ni protecteur ni partisan" (Abdel Aziz Z. , 2009, p. 264)

F: " Ils jurent par Allah qu'ils n'ont pas dit (ce qu'ils ont proféré) alors qu'en vérité ils ont dit la parole de la mécréance [...] Ils n'auront sur terre ni allié ni secoureur " (LeNOBEL C. , 1420 Héjrie, p. 199)

H: " Ils jurent Dieu qu'ils ne l'ont pas dite, alors qu'en vérité ils l'ont dite, la parole de mécréance [...] et ils n'auront sur terre ni patron ni secoureur" (Hamidullah M. , 1989, p. 199)

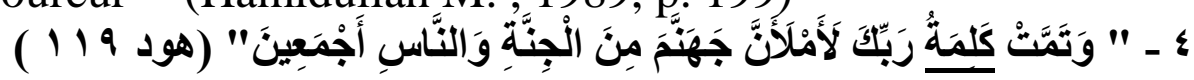

$\underline{\text { Z: }}$ "Et c'est pour cela qu'Il les A Créés. Et la parole de ton Seigneur s'est accomplie: « Je Remplirai sûrement la Géhenne des djinns et des hommes en totalité " (AbdelAziz Z. , 2009, p. 301)

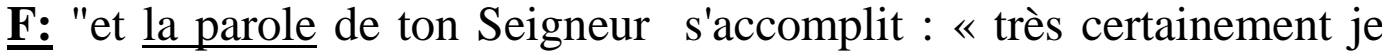
remplirai l'Enfer de djinns et d'hommes tous ensemble " (LeNOBEL, , 1420 Héjrie, p. 235 )

H: "Et la parole de ton Seigneur s'accomplit- : «Très certainement, j'emplirai la Géhenne de djinns et d'hommes, tous " (Hamidullah M. , 1989, p. 235)

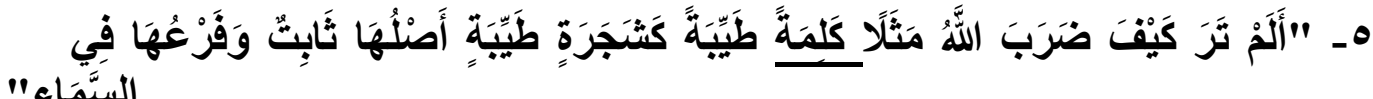

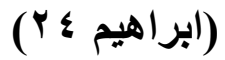

$\underline{\text { Z: }}$ "N'as-tu pas vu comment Allah A Fourni une parabole ? Une bonne parole est comme un arbre bon : sa racine est stable et sa ramure est au ciel." (AbdelAZiz, 2009, p. 327 )

F: "N'as-tu pas vu comment Allah propose en parabole: une bonne parole pareille à un bel arbre dont la racine est ferme et la ramure s'élançant dans le ciel?" (LeNOBEl, 1420 Héjrie, p. 258)

H: "N'as-tu pas vu comment Dieu frappe en exemple la parole excellente? Elle ressemble A un arbre excellent dont la racine est ferme et la ramure, dans le ciel ; " (Hamidullah, , 1989, p. 258) 


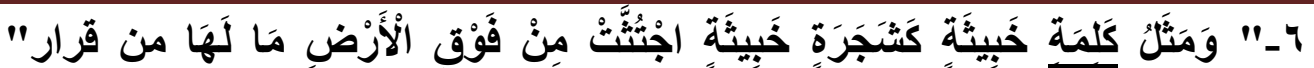

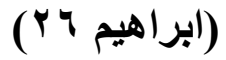

$\underline{Z}$ : "Et la semblance d'une mauvaise parole est comme un arbre mauvais, qui fut arraché de sur la terre, qui n'a nulle stabilité " (AbdeAziz, 2009, p. 327 ).

F: "Et une mauvaise parole est pareille à un mauvais arbre, déraciné de la surface de la terre et qui n'a point de stabilité " (LeNOBEL, , 1420 Héjrie, p. 259).

H: "Et il en est d'une parole mauvaise comme d'une plante mauvaise déracinée de la surface de la terre: elle n'à point de stabilité " (Hamidullah, , 1989, p. 259).

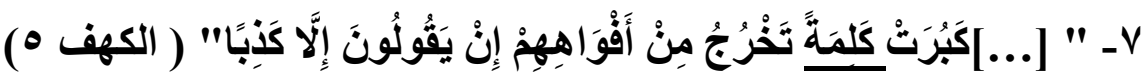

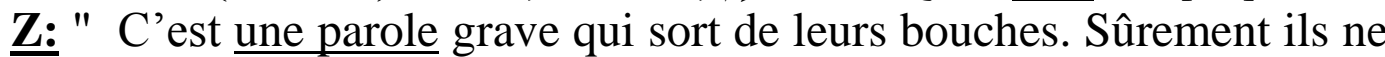
disent que du mensonge" (AbdelAziz, 2009, p. 368).

F: "Quelle monstrueuse parole que celle qui sort de leurs bouches ! Ce qu'ils disent n'est que mensonge" (LeNOBEL, , 1420 Héjrie, p. 294 )

H: " Quelle énormité que la parole qui sort de leurs bouches ! Ils ne disent que mensonge!" (Hamidullah, , 1989, p. 294 )

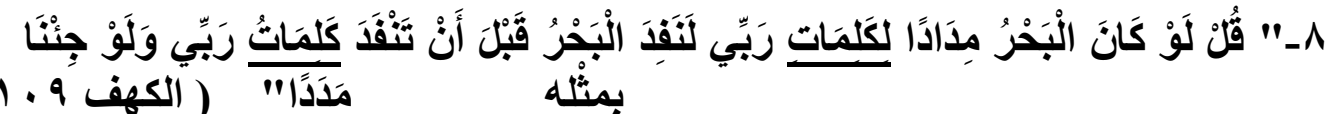

$\underline{\text { Z: }}$ "Dis:« Si la mer était de l'encre pour les paroles de mon Seigneur, la mer s'épuiserait avant que les paroles de mon Seigneur ne s'épuisent, même si nous apportions son semblable comme approvisionnement " (AbdelAziz, , 2009, p. 380)

F: "Dis : "Si la mer était une encre [pour écrire] les paroles de mon Seigneur ,certes la mer s' épuiserait avant que ne soient épuisées les paroles de mon Seigneur, quand même Nous lui apporterions son équivalent comme renfort " (LeNOBEL, , 1420 Héjrie, p. 304 )

H: "Dis: «Si, pour les paroles de Dieu, la mer était encre, certes la mer s'épuiserait avant que ne soient épuisées les paroles de mon Seigneur. » Et quand même Nous en ferions venir en renfort un semblable " (Hamidullah, , 1989, p. 304)

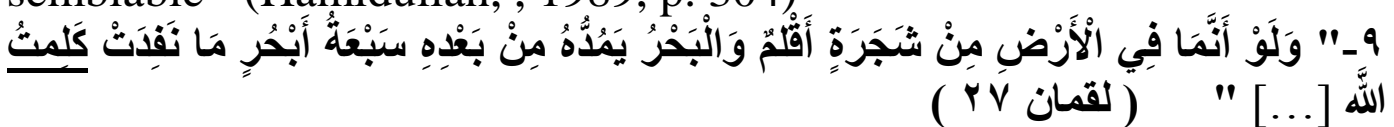

$\underline{\text { Z: }}$ "Si ce qu'il avait sur terre comme arbre était des calames,et que la mer était approvisionnées, après son tarissement,de sept autres mers,

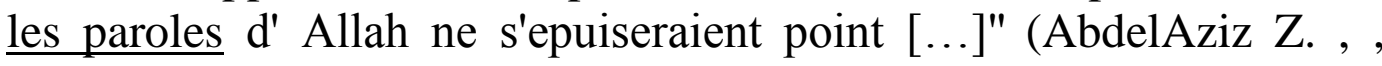
2009, p. 506).

F: "Quand bien même tous les arbres de la terre se changeraient en calames [plumes pour écrire], quand bien même l'océan serait un 
océan d'encre Où conflueraient sept autres océans, les paroles d'Allah ne s'epuiseraient pas [...] "(LeNOBEL, 1420 Héjerie, p. 413) H: "Oui, et si tout ce qu'il ya d'arbres sur terre devenait plumes, et que la mer, et sept mers après elles, fournissent l'encre, les paroles de Dieu ne seraient pas épuisées [...]" (Hamidullah, , 1989, p. 413)

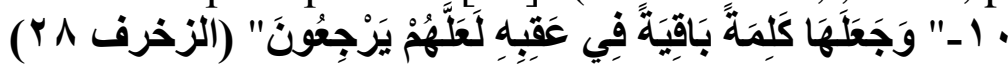

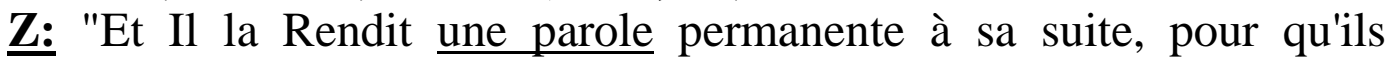
reviennent. " (AbdelAziz Z. , , 2009, p. 491)

F: "Et il en fit une parole qui devait se perpétuer parmi sa descendance peut- être reviendront-ils ?" (LeNOBEL C. , 1420 Héjrie, p. 491 )

H: "Et de ceci Il fit une parole qui devait durer à travers sa descendance. Peut- être reviendraient- ils? " (Hamidullah, , 1989, p. 491)

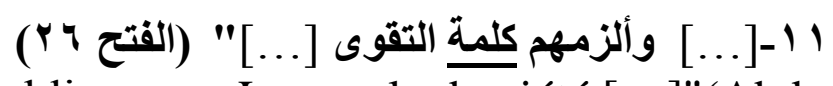

$\underline{\mathbf{Z}}:$ "[...] et leur fit une obligeance: La parole de piété [...]"(AbdeAziz, 2009 , p. 626 )

F:"[...] et les obligea à une parole de piété [...] " (NOBEL, 1428 Héjrie, p. 514)

H: " [...] et les obligea à une parole de piété [...]." (Hamidullah, , 1989, p. 514)

\section{- La correspondance de l'énoncé dans sa traduction:}

Dans cette section, tous les traducteurs choisissent le terme/ l'énoncé (parole) dans leurs traductions, il s'agit de la correspondance de ce mot entre le français et l'arabe.

Dans le verset (1), le terme sلمة est mentionné deux fois, en singulier et en pluriel. Les trois traducteurs se sont contentés de traduire ce terme par (la parole ou ses paroles). En général, la parole signifie un langage prononcé afin de communiquer avec les autres.

D'après les interprètes et linguistes du Coran, le sens du terme (كلمة) en singulier et en pluriel exprime les décrets de la religion de l'Islam. Donc, la signification de l'énoncé dans ce verset la parole de ton Seigneur et (non le mot) " qu'Allah a montré à toutes les religions, même si les polythéistes détestent, et c'est ce que l'on entend par sa perfection, mais le sens de "véracité et justice" est que le Coran est vrai dans toutes les paroles [...] (Pas de changeur pour ses mots) car c'est vrai et correct" (Mughniyah M. J., , 2009, p. 252) .

Aussi le sens est la constitution ou les enseignements de la religion que le Coran ou l'Islam contient de (كلماته) les ordres, décisions et les jugements d'Allah. L'autre interprétation dit :"La parole de ton Seigneur a été faite signifie les jugements prises et les 
dates déterminés par Allah et Il ne les changera jamais" (Al-mahali \& Al-suyouti, , 2001, p. 182).

Dans le verset (2), les traducteurs comptent sur les compléments du nom différents qui suivent le terme(كلمة) afin de déterminer et distinguer entre les deux sens contradictoires: (كلمة اللَّنَينَ كَفَرُوا) كلمة). L'aspect interprétatif dit: "La Parole d 'Allah signifie Le monothéisme d 'Allah. Alors que la parole des mécréants signifie l'infidélité et le polythéisme" (Mughniyah, 2009, p. 45). L'interprète Mughniyah a comparé dans son exégèse entre deux parties: la première représente la perte et la déception, tandis que la seconde incarne tout ce qui est bon et réussi. Jalal Al-Din ajoute que: "La parole de ceux qui ne croient pas est un appel à Partenariat en Allah (le bas) et le sens (et la parole d'Allah) qui prédomine" (Al-mahali \& Al-suyouti, 2001, p. 247). La parole des mécréants veut dire un appel pour le mal cependant la parole d'Allah exprime l'énoncé victorieuse du Monothéisme en Dieu.

Puisque le verset (3) contient le verbe ( قال Qalâ) signifiant (Dire, parler, proférer ou prononcer), les trois traducteurs ont réussi à traduire le terme (La parole) précédé de verbe (dire) ou (proférer) pour exprimer le terme (كلمة). Pour bien évaluer la qualité de ces trois traductions, On a besoin de connaitre les conditions et la raison de la descente de ce verset: "Ce verset concerne Abdullah bin Abi, deux hommes luttant contre l'un d'eux Jahni et l'autre est Ansari. Jahni défait le Ansari. Abdullah a demandé ses partisans, soutenez-vous votre frère? En ajoutant : Par Allah, notre relation avec Muhammad est comme le proverbe disant: "Nourris ton chien pour il te mangera" (Al-Damashqi, 1990, p. 355).

Certains hypocrites ont parlé de manière inacceptable du Prophète (Muhammad) et de l'Islam, et Cette insulte au Messager d'Allah reflète une foi. L'interprétation de Mughniyah: "Le pronom de verbe jurer cite un groupe d'hypocrites qui ont prononcé une expression d'infidélité sur le Messager d'Allah Muhammad. Quand il leur a demandé, ils ont craint et ont juré, Dieu leur a menti" (Mughniyah, 2009, p. 70). L'interprétation Al-Jalaleen, a soutenu l'explication précédente:"(Ils jurent) c'est à dire les hypocrites (Par Allah qu'ils n'ont pas dit) ce que vous avez été informé de leur insulte (Ils ont dit le terme d'incroyance et ils ont montré l'incroyance après avoir montré l'Islam)" (Al-mahali \& AL-suyouti, , 2001, p. 253) .

La locution (كلمة ربك), qui a un sens de la (parole d'Allah) est un avertissement, une menace ou une promesse et un engagement du Seigneur pour les hommes. D'après l'interprétation:"Allah remplira l'enfer des désobéissants des djinns et des hommes" (Mughniyah, 2009, p. 279 ). Tafseer al-Jalaleen dit que l'interprétation de terme est expliquée dans la suite du verset: " Je remplirai 
l'enfer des djinns et des gens Tout ensemble" (Al-mahali \& Alsuyouti, 2001, p. 302). En résumé, ce terme nous confirme la pleine connaissance et la sagesse d'Allah, puisqu'Il est le Créateur, Il connait d'avance qui mérite le paradis, et l'autre qui mérite l'enfer.

Dans le verset (5) le $\mathrm{Z}$ et le $\mathrm{F}$ ont utilisé (une bonne parole); mais le $\mathrm{H}$ a recours à renforcer l'énoncé par l'adjectif (excellent). L'interprétation de la locution (الكلمة الطيبة):" La bonne parole signifie la parole du monothéisme et de la foi, la parole de la malice veut dire l'infidélité et l'athéisme" (Mughniyah, 2009, p. 442). C'est la parole de l'individualiste d'Allah et de la foi ou c'est la parole de la vérité. L'autre interprétation explique le terme (الكلمة الطيبة) : " Il n'y a pas d'autre Dieu qu'Allah" (Al-mahali \& Al-suyouti, , 2001, p. 303). Donc le discours a fait ressembler (le bon mot) (الكلمة الطيبة) au bon arbre, alors que, dans le verset (6), Il décrit (le mot d'incrédulité) comme "l'arbre de la méchanceté" détaché et n'a aucune racine car il n'a pas de profondes et pas de branches ascendantes et aucune bénédiction. C'est le cas de l'incroyant qui n'y a pas de stabilité et il n'a aucune de bonnes actions offertes à Allah.

Dans le verset (7), le nom ou l'attribut associé à ce terme a joué un rôle important dans la détermination de sa signification précise. Le (Z) a ajouté l'adjectif (grave) pour montrer la gravité de ce mot. Le (F), pour la même raison a décidé d'utiliser, avant le terme (parole) l'énoncé (Quelle monstrueuse).

Le $(\mathrm{H})$ a exprimé la méchanceté du ce vocabulaire par mettre l'adjectif indéfini (Quelle) avec le nom (énormité). Selon le côté explicatif, Mughniyah dit: " Rien n'est plus dangereux que de mentir, et le plus grave est la calomnie d'Allah et la prétention c'est Celui qui prive et permet sans avoir aucune preuve. Le mensonge le plus dangereux c'est la prétention que le fils est le sien " (Mughniyah, 2009, p. 102). Le mensonge est dangereux, mais dire des mensonges sur Dieu est le plus dangereux. Toutefois, le plus dangereux est de dire qu'Allah a un fils. Une autre interprétation dit :" Quel mot dangereux qu'ils ont prononcé (qui est destiné à dénigrer) " (Almahali \& Al-suyout, , 2001, p. 381).

D'autre part, Tafseer Al-Jalaleen explique que l'homme qui a dit ce terme est inconnu. L'interprétation de ce terme est de dénoncer ce qu'a été dit. Pour plus d'informations sur ce verset, l'histoire dit qu'Allah a mentionné que certaines personnes prétendent qu'Allah a un fils. Allah répond dans ce verset pour nier cette croyance dangereuse. Alors la parole signifie (une croyance) mais c'est une mauvaise croyance.

En ce qui concerne la traduction de verset (8), le terme (كلمة) se trouve deux fois en pluriel (كلمات ربي).Le point de vue était basé sur les interprétations de ce verset: "La signification de terme (كلمات ربي) dans 
ce verset ne signifie pas la parole composée d'alphabets mais la capacité de créer des créatures quand Dieu le veut " (Mughniyah, 2009, p. 166). Cette expression-là a deux satins. Elle possède un sens vrai et une signification métaphorique. La capacité d'Allah de créer des objets est une competence qui n'a pas de fin. La deuxième explication dit: " Les paroles de Dieu veulent dire ses sagesses et ses merveilles " (Al-mahali \& Al-suyouti, , 2001, p. 395) .Cela confirme qu'Allah, le Créateur est capable à engendrer infiniment sa parole même si est une propriété de l'être humain. Ce dernier ne peut pas le faire.

Également nous constatons dans le verset (9) que les trois traducteurs sont $\mathrm{d}^{\prime}$ accord à utiliser le terme (les paroles) en considérant (كلمات الله) comme (Le propos d' Allah). L'interprétation explique ce terme: "Sept mers indiquent la multitude et les paroles d'Allah signifient sa capacité à créer des créatures quand Il veut et que la capacité d'Allah n'a pas de limites" (Mughniyah, , 2009, p. 167 ). L'autre livre d'interprétation dit: "La signification de (كلمات الله dans ce verset est les informations d'Allah écrites avec sept mers d'encre même s'Ilya plus de mers" (Al-mahali \& Al-suyouti, , 1989, p. 543).

Afin d'éclaircir le sens dans le verset (10), le (Z) a mis l'adjectif permanent, cependant que le $(\mathrm{F})$ et le $(\mathrm{H})$ ont utilisé le pronom relatif qui pour expliquer l'effet du sens du terme (mot).

L'interprétation nous dit: "Le terme (mot) signifie le mot monothéisme" (Mughniyah, , 2009, p. 544). Abraham, le prophète, a recommandé que ses fils respectent le Monothéisme en Allah et agissent selon ses enseignements, et si l'un de ses autres fils le nie, on le lui rappellera. Al-Jalaleen a un avis déférent :"La signification $d u$ terme (كلمة باقية) exprime la génération d'Abraham dont certains croient toujours en l'unicité d'Allah c'est-à-dire que les gens de La Mecque laissent l'incrédulité en adorant, à nouveau, la religion d'Abraham" (Al-mahali \& Al-suyouti, , 2001, p. 649).

Dans le verset (11), les traductions du terme (كلمة) sont similaires. Les traducteurs considèrent le terme (la parole de piété) ou (une parole de piété) comme le meilleur moyen de transmettre l'idée du terme (كلمة الثقوى) au place de (le mot de piété) : "Le sens du terme (كلمة التقوى )'est qu'il ny a de Dieu que Allah" (Mughniyah, , 2009, p. 99). Le sens le plus probable de ce mot est d'agir avec piété. L'autre interprétation était en faveur de la première interprétation: "Dans ce verset, le sens de terme (كلمة التقوى) Il n'y a pas d'autre Dieu qu'Allah" (Al-mahali \& Al-suyouti, , 2001, p. 683 ). Les interprètes s'accordent sur l'interprétation du terme dans ce verset. On conclue cette section par l'accord des traducteurs sur la traduction du terme sلمة par (parole). 
Le schéma suivant montre que les traductions de 33 versets en total de terme (كلمة) sont accordées au terme (parole):

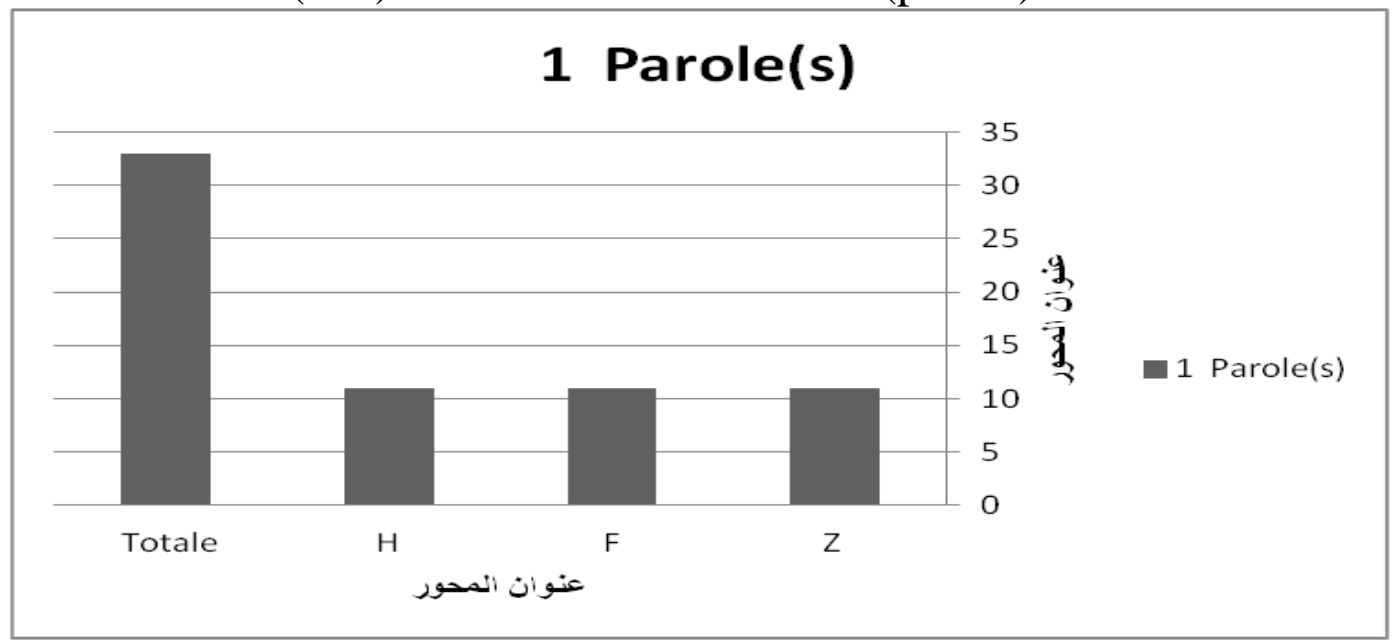

- Deuxième section : le désaccord de la traduction

Après avoir présenté des versets dans lesquels les traducteurs ont utilisé un mot synonyme du terme (كلمة), on va aborder dans cette section les 11 autres versets dans lesquels les traducteurs ont recours

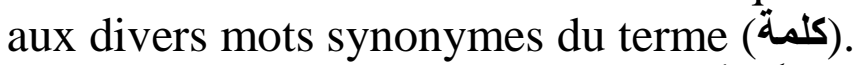

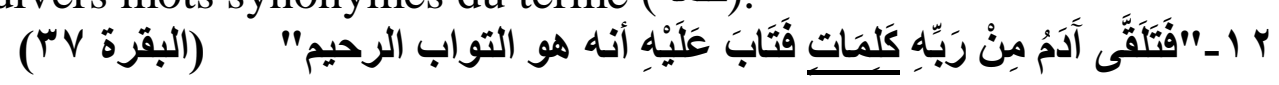
Z: "Puis Adam reçut de son Seigneur quelques mots, et par là Il lui $\mathrm{A}$ Fait Rémission. Il Est, Lui, le » Rémissif, le Miséricordieux" (Abdel Aziz Z. , 2009, p. 64).

F: "Puis Adam reçut de son Seigneur des paroles, et Allah agréa son repentir car c'est lui certes l'Accueillant au »repentir, le Miséricordieux." (LeNOBEL C. e., 14201 Héjrie, p. 6).

H: "Puis Adam reçut de son Seigneur des paroles. Puis Dieu accueillit son repentir. Il est le pardonner, le Miséricordieux vraiment" (Hamidullah M. , Le Saint Coran, 1989, p. 6).

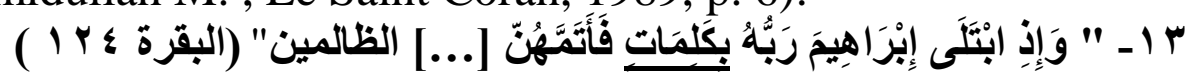

$\underline{\text { Z: " }}$ "Et lorsque Abraham fut éprouvé par son Seigneur, par des Commandements qu'il accomplit [...]" (Abdel Aziz Z. , 2009, p. 78)

F: " [Et Rappelle-toi] quand ton seigneur eut éprouvé Abraham par certains commandement. Et qu'il les eut accomplies [...] " (LeNOBEL C. , 1420 Héjrie, p. 19)

H: «Et Abraham!...Quand son seigneur l'eut éprouvé par certaines paroles et qu'il les eut accomplies[...] " (Hamidullah \& Muhammad, , 1989, p. 19)

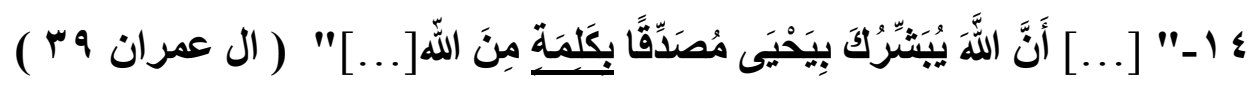

$\underline{\text { Z: }}$ ::[...] «Allah te Fait l'annonce de Jean, croyant en une parole venant d'Allah [...]". (Abdelaziz, 2009, p. 115) 
F: " [...] voilà que Allah t'annonce la naissance de Yaya, confirmateur, d'une parole d'Allah [...]" (LeNOBEL C. , 1420 Héjrie, p. 55)

H: " [...] voilà qu'Allah t'annonce Jean, confirmateur d'un verbe de Dieu .[...]." (Hamidullah, , 1989, p. 55)

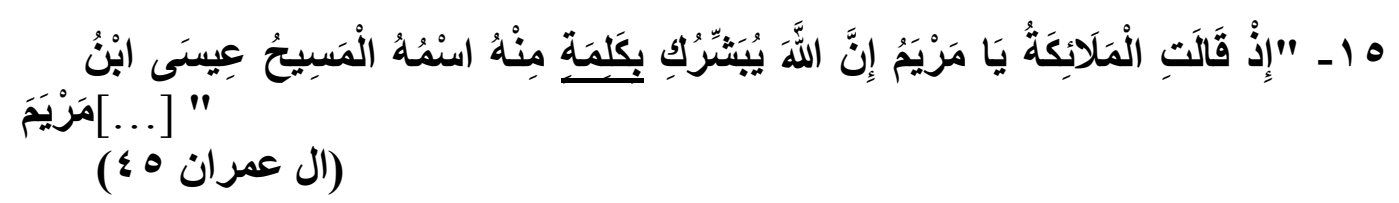

$\underline{\mathbf{Z}}$ : "Lorsque les Anges dirent: «O Marie, Allah te Fait l'annonce d'une parole de Sa part : il s'appelle le Messie, Jésus fils de Marie [...]" (AbdelAziz, 2009, p. 115).

F: " (Rappelle -toi) quand les anges lui dirent: «O Marie, voilà qu'Allah t'annonce une parole de sa part : son nom sera« alMasih»«Issa» fils de Marie [...]" (LeNOBELCORAN, 1420 Héjrie, p. 55 )

H: "Quand les Anges dirent : O Marie Voilà qu'Allah t'annonce un verbe de sa part: son nom est l'oint, Jésus fils de Marie [...]." (Hamidullah, 1989, p. 55).

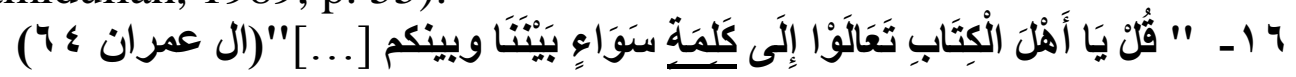

$\underline{\text { Z: }}$ "Dis: «O gens du Livre, venez-en à une parole normative entre nous et vous [...]" (Abdel Aziz , 2009, p. 118)

F: "Dis: «O gens du Livre, venez à une parole commune entre nous et vous. [...] "(Le NOBEL CORAN, 14201 Hejrie, p. 58)

H: "Dis : «O gens du Livre, venez-en à un dire qui soit commun entre nous et vous. [...]" (Muhammad Hamidullah , 1989, p. 58)

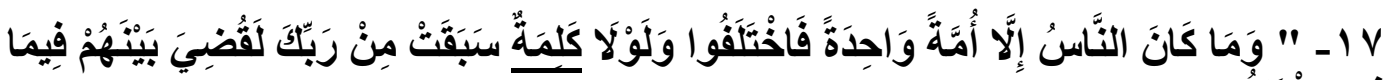

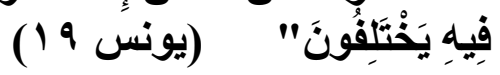

$\underline{\mathbf{Z}}$ : "Les hommes ne formaient qu'une seule communauté, mais ils divergèrent. Et n'était-ce un Décret préalable de ton Seigneur, c'en aurait été fait entre eux sur ce dont ils divergeaient" (Abdel Aziz z., 2009, p. 275)

F: "Les gens ne formaient(à l'origine) qu'une seule communauté, Puis ils divergèrent. Et si ce n'était-ce une Décision préalable de ton de ton Seigneur, les litiges qui les opposaient auraient été tranchés " (CORAN, 1420 Héjrie, p. 210).

H: "Les gens n'étaient qu'une seule communauté .Puis ils divergèrent .Or, n'était qu'une parole de Ton Seigneur eût pris les devants, c'en aurait été fait, entre eux, de ce en quoi ils divergeaient! " (Hamidullah M. , , 1989, p. 210)

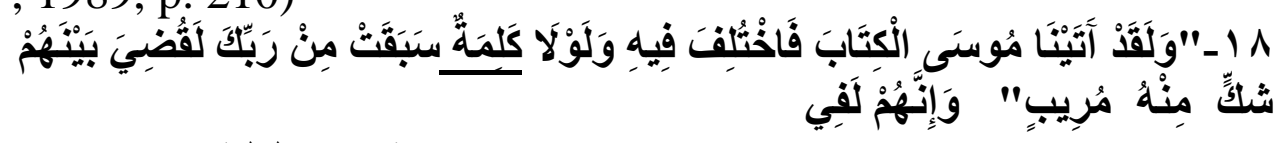


$\underline{\text { Z: }}$ "Et Nous Donnâmes le Livre à Moïse, il y eut alors divergence à son sujet. Ne fût-ce un Décret préalable de ton Seigneur, c'en aurait été fait parmi eux. Ils en sont sûrement dans un doute inquiétant à son sujet" (Abdel Aziz Z. , 2009, p. 300)

F: "Et Nous avons déjà donné à Moïse le Livre. Il y a eut des divergences à son sujet. S'il n'y avait pas un décret préalable de la part de ton Seigneur, tout aurait été décidé entre eux. Et ils sont, à ses sujets pleins d'un doute troublant" (Le NOBEL C. , 1420 Héjrie, p. 234)

H: "Et très certainement. Nous avions donné à Moïse le Livre. Puis, on y divergea. Et, n'était qu'une Parole de la part de ton Seigneur eût pris les devants, tout aurait été décidé, entre eux ! Oui, ils sont à son sujet, en un doute qui mène à l'incertitude. " (Hamidullah M. , 1989, p. 234).

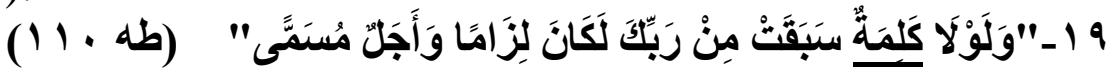

$\underline{\text { Z: }}$ "Et n'était-ce un Décret préalable de ton Seigneur, cela aura été une obligation et un terme fixé. " (AbdelAziz, , 2009, p. 401 ).

F:"N'eussent- été un décret préalable de ton Seigneur et aussi un terme déjà fixé, (leur châtiment) aurait été inévitable (et immédiat)" (LeNOBEL, 1420Héjrie, p. 321 ).

H: "N'était qu'une parole de ton Seigneur eût pris les devants, et aussi un terme dénommé, cela leur collerait!" (Hamidullah, , 1989, p. 321).

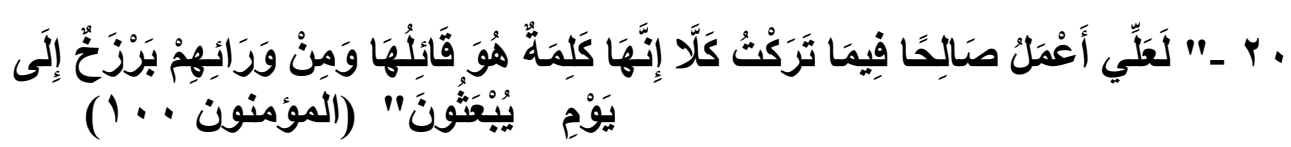

$\underline{\mathbf{Z}}$ : "Il se peut que je fasse œuvre méritoire en ce que j'ai délaissé. » Sûrement pas! Ce n'est qu'un mot que lui a dit. Et de derrière eux un intervalle jusqu'au jour où ils seront ressuscités " (AbdelAziz, , 2009, p. 432)

F: "Afin que je fasse du bien dans ce que j'ai délaissées »Non c'est simplement une parole qu'il dit. Derrière eux, cependant. il y a une barrière, jusqu'au jour où ils seront ressuscités" (LeNOBEL, 1420 Héjrie, p. 348 )

H: "Peut -être ferai je du bien, dans ce que je délaissais! » Non, non! Cela, c'est une parole qu'il dit. Derrière eux, cependant, il y a le monde intermédiaire, pour jusqu' au Jour où ils seront ressuscités" (Hamidullah, , 1989, p. 348 )

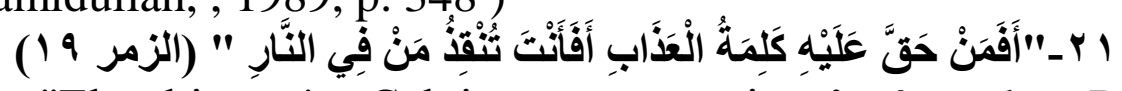

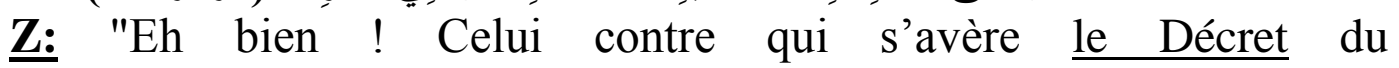
châtiment...délivrerais-tu, toi, celui qui est dans le Feu?! " (AbdeAziz, 2009, p. 564 )

F:" Eh bien, quoi !celui contre qui s'avère le décret du châtiment,... est-ce que tu sauves celui qui est dans le Feu ? "(LeNOBEL, 1420 Héjrie, p. 460) 
H: "Eh bien, quoi !celui contre qui s'avère la parole du châtiment, quoi! peux -tu sauver celui qui est dans le feu ?" (Hamidullah, , 1989, p. 460$)$.

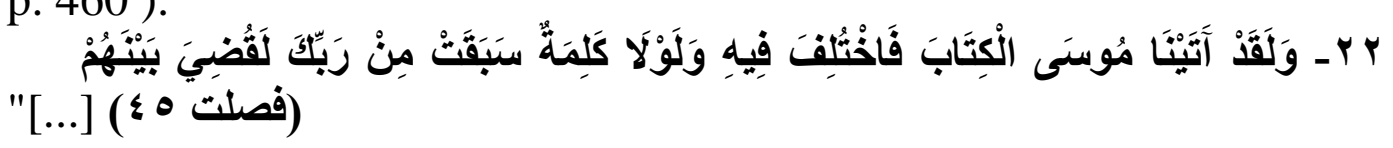

$\underline{\text { Z: }}$ "Et Nous Donnâmes le Livre à Moïse, alors il y eut divergence à son sujet. Et ne fût-ce un Décret préalable de ton Seigneur, c'en aurait été fait parmi eux. [...] "(AbdelAziz Z. , , 2009, p. 587)

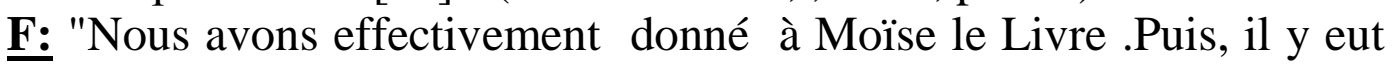
controverse là -dessus. Et si ce n'était une parole préalable de ton Seigneur, on aurait certainement tranché entre eux. [...]" (LeNOBEL, , 1420 Héjrie, p. 481)

H: "Et très certainement Nous avions apporté à Moïse le Livre. Puis, il y eut divergence. Or si de la part de ton Seigneur une parole n'eût pas pris les devants, tout aurait été décidé entre eux [...]" (Hamidullah, , 1989, p. 481).

\section{- L'équivalence de l'énoncé dans sa traduction:}

Dans ces trois traductions, pour exprimer le sens du vocabulaire (كلمات) le (Z) recours à traduire littéralement ce terme par (quelques mots) dans le verset (12); cependant que le (F) et le (H) ont un autre point de vue car ils ont imaginé un dialogue ou une conversation entre Allah et son prophète. Or, ils ont choisi (des paroles) qui signifient (des propos).

L'interprétation du verset dit :"Adam a ressenti la catastrophe, il a regretté en demandant sincèrement à son Seigneur d'accepter son repentir et Il l'a accepté" (Mughniyah, , 2009, p. 84). Cela signifie que quand Adam a commis un grand péché, il avait des remords et il a demandé à son Seigneur sincèrement d'accepter Son repentir. Allah lui a pardonné. L'interprétation Al-Jalaleen a soutenu le point de vue précédent :" Seigneur les lui a inspirés, avec les quels Adam a prié et Il a accepté sa repentance " (Al-mahali \& Al-suyout, 2001, p. 9). Allah a enseigné à Adam ces mots pour lui permettre de demander le pardon, puis Il lui a pardonné.

Les traducteurs $(\mathrm{Z})$ et $(\mathrm{F})$ ont pu atteindre la signification précise du terme (كلمات) dans le verset (13), puisqu' ils ont préféré l'énoncé (Des Commandements) qui signifie les règles de conduite édictée par l'autorité d'Allah et les exigences à remplir pour gagner la satisfaction d'Allah. Le traducteur $(\mathrm{H})$ avait une autre vue sur la traduction de ce terme, il l'a traduit superficiellement et ne pas traiter le contexte: pour lui, le terme juste est (certaines paroles).

Dans l'aspect de l'interprétation:" Allah tout puissant qu'il a ordonné à Ibrahim des devoirs comme le sacrifice de son fils " (Mughniyah, 2009, p. 195). Le terme (كلمات), veut dire les 
commandements et les interdictions, y compris l'ordre d'Allah à son prophète Abraham pour égorger son fils, le prophète a répondu à tous ces ordres. L'autre explication: "Les ordres sont les rituels du pèlerinage, le rinçage inhalation, cure-dents, couper les moustaches et les ongles et peigner les cheveux" (Al-mahali \& Al-suyout, 2001, p. 26) .

Par ailleurs, dans les versets (14 et 15), le (Z) et le (F) utilisent le terme (une parole) envoyée par les anges .On pense que les deux traducteurs n'ont pas abordé l'éloquence du Coran car ils n'ont pas cherché profondément le sens caché. En effet, en traduisant (كلمة) par (un verbe), le $(\mathrm{H})$ a traité ce terme professionnellement car il était relativement plus clair que l'utilisation du mot "verbe" qui montrerait la grande puissance du Seigneur pour exécuter directement sa volonté (Allah a créé le prophète Jésus avec un seul mot). A propos de l'interprétation :" La parole d'Allah est une référence à Jésus, qu'Allah a créé par le verbe (sois) sans père" (Mughniyah, 2009, p. 53). Grâce au verbe (être), la capacité et la volonté d'Allah se représentent par le miracle de la création de (Jésus) sans père. AlSuyouti est d'accord avec l'interprétation précédente: "Mentionne (Les anges ont dit), c.-à-d. Jibril (Marie, qu'Allah vous dira un mot de Lui), c.-à-d., Un enfant" (Al-mahali - Al-suyouti , 2009, p. 71). Afin de confirmer l'opinion favorable à la troisième traduction, Il y a une expression dit: "Le verbe s'est fait chair" (Sabek, 1972, p. 1216).

L'utilisation dans les trois traductions (une parole) et (un dire) nous montre dans le verset (16) que les traducteurs n'ont pas atteint le sens requis. Le terme (كلمة) signifie «un accord». Les trois traductions sont littérales parce que ce verset contient des instructions du Seigneur à Son Messager sur la façon d'agir avec les adeptes d'autres religions. Le $(\mathrm{Z})$ et le $(\mathrm{F})$ ont mis (une parole normative) et (une parole commun) au lieu du terme (كلمة سواء) cependant que le $(\mathrm{H})$ a préféré utiliser l'expression (un dire qui soit commun) pour traduire ce terme.

D'après l'interprétation, "Allah Tout-Puissant a dit: O Mohammed ne discute pas avec eux, mais suis, avec eux, cette approche que chaque personne rationnelle témoigne de la vérité. Voire c'est la raison et la conscience en les appelant à ce que l'e et les livres célestes l'ont tous approuvé pour adorer un Dieu sans aucun partenaire" (Mughniyah , 2009, p. 81). Allah demande au Prophète Muhammad de négocier avec les partisans d'autres religions afin de parvenir à un accord sur des points communs entre eux. Une autre interprétation appuie l'interprétation ci-dessus :" La doctrine qui doit être convenue de (Adorer Dieu seulement et il n'y a pas de partenaire et ne pas considérer certains d'entre nous des dieux au lieu de Allah)" (Al-mahali \& Al-suyouti, 2001, p. 75). Après avoir consulté ces 
deux interprétations, on comprend que le terme (كلمة) signifie un accord.

Ainsi, les traducteurs ne sont pas d'accord dans les versets (17 et 18) sur ce terme (كلمة). Le (Z) et le (F) ont réussi à atteindre le sens le plus précis ,ils l' ont mis le terme (un décret) et (une décision), mais le $(\mathrm{H})$ a traduit ce terme telle qu'elle est en se contentant de traduire le terme ( كلمة par (une parole).

Le point de vue interprétatif voit que : "La signification de la parole de Dieu, ici, ne pas hâter la punition des infidèles"

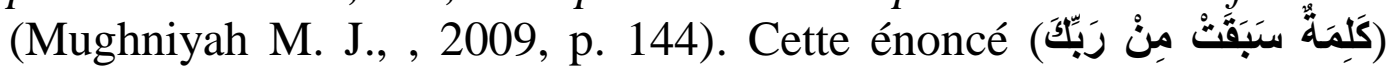
signifie un ordre d'Allah de retarder punir les dés obéissants. Cependant l'autre interprétation dit:" C'est l'ajournement du tourment au Jour de la Résurrection "(Al-mahali \& ALsuyouti, , 2001, p. 268). En d'autres termes, Allah a déjà promulgué un décret de ne pas périr les gens que après l'expiration de leurs termes, Il enverra les incroyants en Enfer et les fidèles au Paradis. Selon Sa décision, Allah retarde la pénalité jusqu' au Jour de la Résurrection.

Afin d'évaluer le niveau la traduction du terme (كَ) dans le verset (19), il est logique et nécessaire de connaître sa signification exacte. Puisque la définition de (un Décret) dit: "décision, volonté d'une puissance supérieure" (Rey, 1998, p. 339) . Le (Z) et le (F), qui l'ont choisi comme un synonyme du terme (كلمة), étaient plus précis dans leurs traductions mais, le $(\mathrm{H})$, qui a utilisé le vocabulaire (une parole), n'a pas traité ce terme profondément.

On consulte les livres d'interprétation, pour étayer ce point de vue: " Le sens de terme (كلمة ) et le destin est le jugement d'Allah Dieu Tout-Puissant d'ajourner le tourment" (Mughniyah, , 2009, p. 253 ).

Dans le verset (20) qui contient une conversation entre Allah et un homme pendant sa mort, le ( $\mathrm{Z}$ ) a mis le terme (un mot) au lieu de (كلمة) et nous pensons que ce genre de traduction n'explique pas l'idée au destinataire cependant que le $(\mathrm{F})$ et $(\mathrm{H})$ ont utilisé (un parole). Lorsque Le (F) a ajouté l'adverbe (simplement) au mot (une parole), a clarifié certaine signification.

Le $(\mathrm{Z})$ et le (F) recours au (le décret) dans le verset (21), qui signifie le pragmatique ou la sanction. C'était un bon moyen de transmettre l'idée tandis que le $(\mathrm{H})$ a recours (la parole) mais, le sens veut dire, dans ce cas (un langage humain articulaire visant à communiquer la pensée). Il est logique que chaque mot prononcé par Allah devenu sa Parole. Cela pour éviter de fouiller dans les détails de la signification du verset.

L'aspect interprétatif de ce verset, comme nous l'a expliqué Mughniyah :" Non, il n'y a aucun moyen de sauver et sauver du tourment de Dieu, sauf le bon travail" (Mughniyah, , 2009, p. 404). Sauf le travail juste et le pardon d'Allah et sa générosité, l'homme ne 
possède pas de manière de la délivrance de l'enfer. Sur le même verset, il y a une autre explication: "Il n'y a aucun moyen de se sauver du châtiment de Dieu, sauf le bon travail juste" (Al-mahali \& Al-suyouti, 200\&, p. 608). Le terme (كلمة) est une promesse et une menace de Dieu disant (je remplirai l'enfer).

Dernier verset dans notre corpus est le (22), on constate qu'il y a une similarité entre le $(\mathrm{F})$ et $(\mathrm{H})$ mais, le $(\mathrm{Z})$ était plus proche de la signification et de l'éloquence du Coran par le signifiant (Le préjugé) : l'interprétation d'Al Tafessir Al kashef montre que :" $L a$ sagesse de Dieu voulait reporter le châtiment des criminels jusqu'au Jour de la Résurrection" (Mughniyah M. J., , 2009, p. 499 ). Le terme veut dire: Allah a ordonné de retarder la punition des pécheurs au jour du jugement. Sans cette décision, tous les tyrans, les pécheurs et les infidèles n'auraient pas la chance de vivre sur terre. La deuxième interprétation soutient l'interprétation précédente: Reporter le châtiment des créatures jusqu'au Jour de la Résurrection "(Al-mahali \& Al-suyouti, , 2001, p. 636) .

On peut conclure le désaccord des traducteurs par le tableau et le schéma suivants :

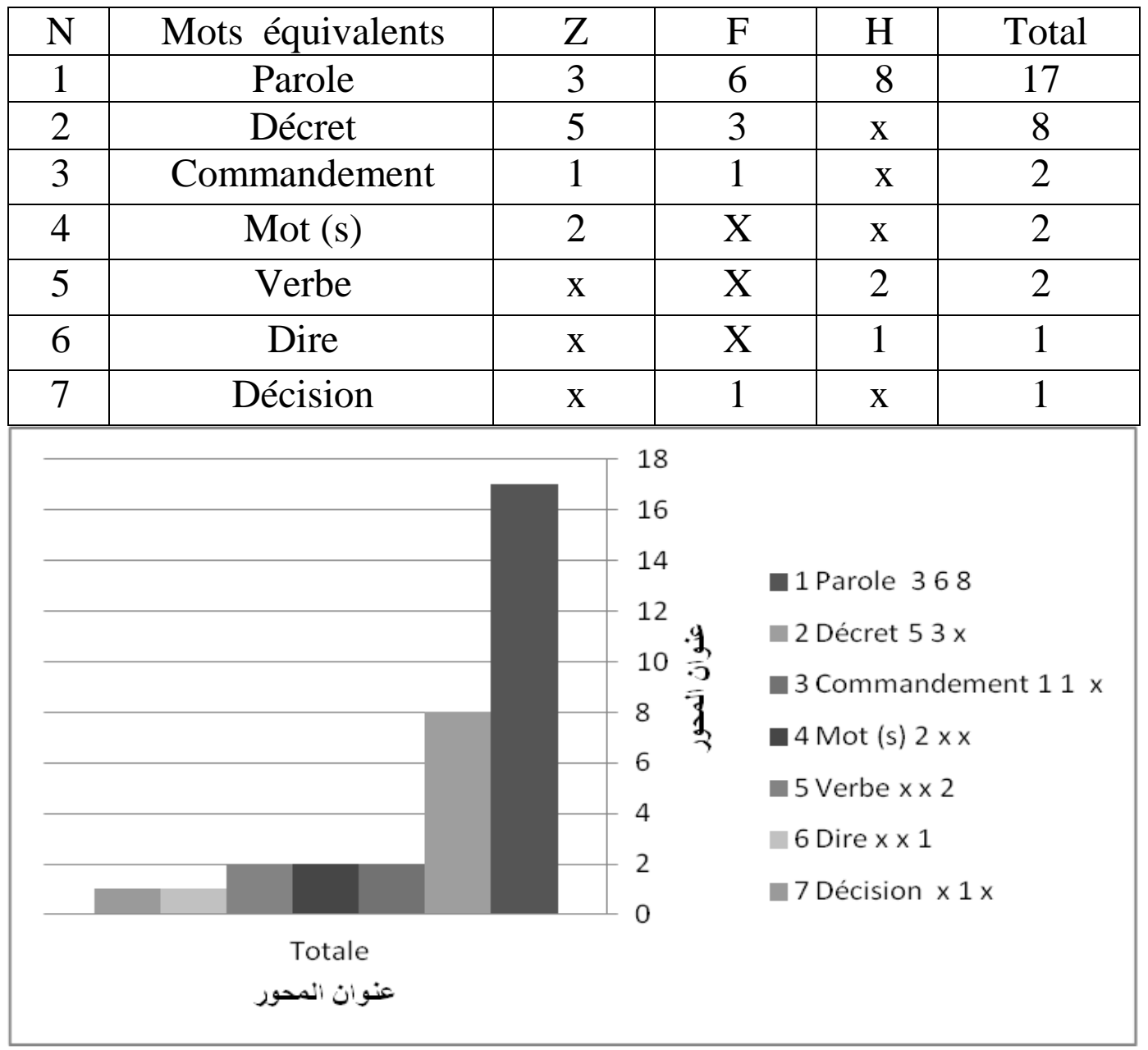


Afin de terminer notre analyse de deux sections, nous conclure les résultats pour les ( 22 versets) par le tableau et le schéma statistiques suivants :

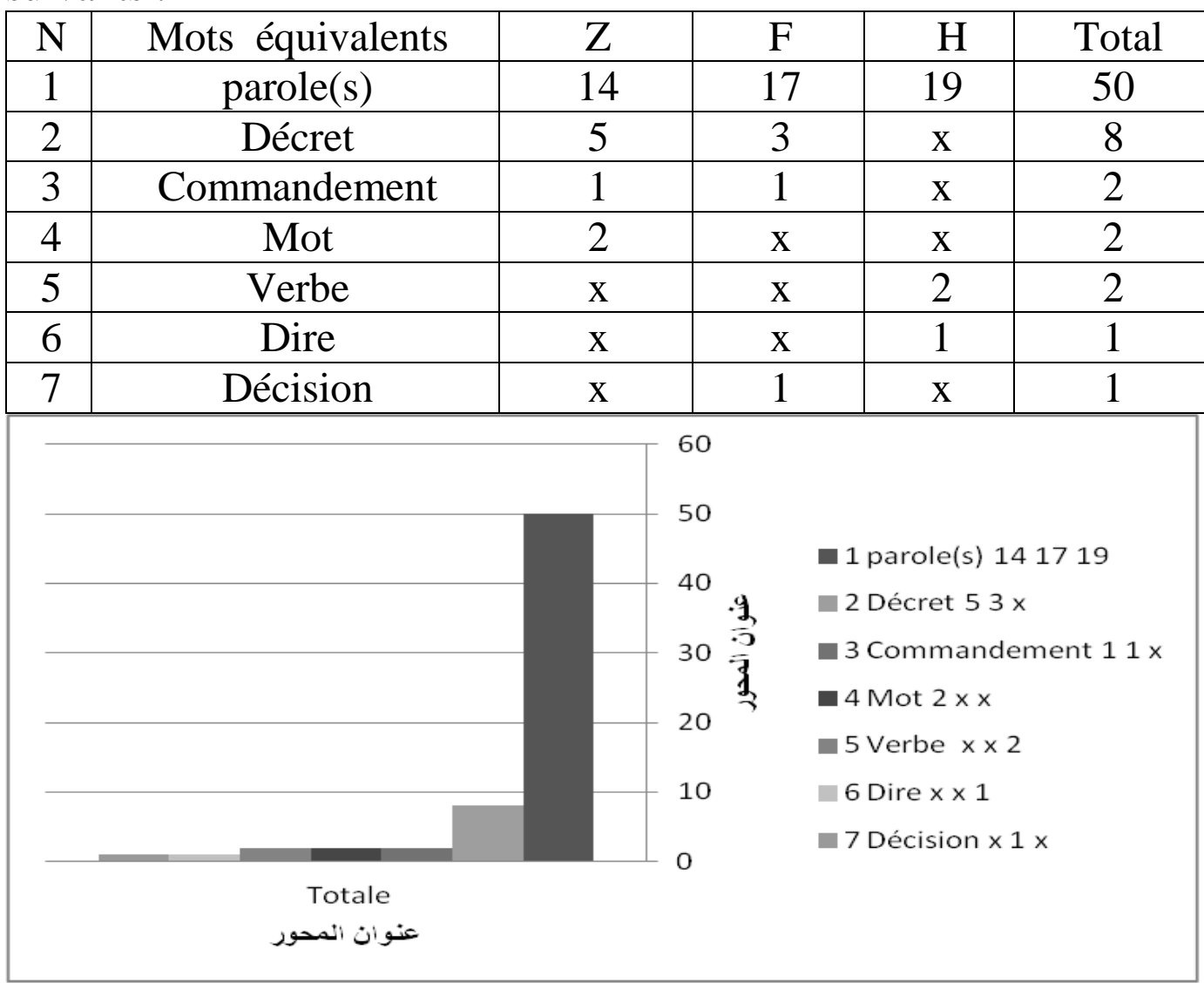

\section{Conclusion}

Il s'agit du choix du traducteur dans sa traduction. La polysémie du mot consiste une contrainte devant l'opération traduite. Autrement dit, quel énoncé doit -il choisir ? Dans cet article les traducteurs, après avoir analysé le corpus, montre bien qu'il y a deux tendances: la correspondance qui reflète la traduction littérale, la deuxième s'agit de l'équivalence qui représente l'interprétation de l'énoncé à traduire.

Il y avait toujours une tendance à traduire beaucoup de livres religieux, recherches ou articles sur ce sujet qui représente une autre difficulté de nombreuses langues même si les traducteurs sont professionnels dans leurs domaines. Mais il reste toujours une difficulté de transmettre la nature et l'éloquence du Coran. Le terme ) (كلمةen singulier ou en pluriel montre une nuance dans son sens coranique.

Les trois traductions sont accordées dans 11 verstes par l'énoncé (parole) comme les tableaux et les schémas nous indiquent dans cet article. Par contre, les trois traductions ne sont pas d'accord sur les autres 11 versets par différentes énoncés comme : mot, parole, décret, décision, verbe, commandement et dire. C'est pour cela et pour 
chercher à la sobriété de cette recherche, on a formé les résultats dans des tableaux et des schémas.

Après avoir abordé la traduction du terme (كلمة) dans 22 versets. Et pour répondre à notre question posée, les trois traductions, qui ont réussi de traduire le mot juste dans la tendance d'équivalence plus que correspondance. Et malgré notre estimation étant mentionnées dans cette recherche, on est sûr que pour obtenir une bonne traduction, il est nécessaire de suivre les recherches sur la question de l'interprétation du Coran afin de permettre au traducteur d'être en conscient au sens exact et correct pour tout le contenu dans le Coran.

En conclusion, on espère que cette simple recherche représente un pas en avant vers cet aspect de la traduction.

\section{Références}

\section{Bibliographie}

ABDEL Aziz Zeinab. Le Coran Traduction du sens de ses Versets. Conveying Islamic Message Society. Alexandrie 2009

ABDEL Latif Zainab. Papiers de recherche en traduction. Maison al Mammoun de la traduction et de la publication Bagdad 2014.

AL -DAMASHQI, I. a.-H.-F.-Q. Tafsir al coran alkarim,V2( Texte arabe). Dar Al jil. Beyrouth 1990.

AL- ZARKANY Mohamed Abdul Azim. Les sourcese de la reconnaissance dans les sciences du Coran, (Texte arabe). Maison Alkitab al araby. Beyrouth 1995.

ALLUSH Jalal al-din ibn Tahir. Les dispositions de la traduction du Coran, (Texte arabe) Dar Ibn Hazm. Beyrouth 2008.

AL-MAHALI Jalal al-Din Muhammad ibn Ahmad 'AlSUYOUTI Jalal Al-Din Abdul Rahman ibn Abi Bakr. Tafseer alJalaleen. (Texte arabe). Maison al-Hadith. Le Caire. 2001.

AL-OTHAIMEEN Muhammad bin Saleh. Explication Riyad Al-Salheen, partie V (Texte arabe). Madar Al Watan de la publication Arabie saoudite. 1429 Héjrie.

ANANI Mohammed. Art de la traduction (Texte arabe). Nashiroun. Beyrouth. 1996.

ARIF Aziz Modèles du défaut dans la traduction du Coran. (Texte arabe). Revu al maouride 4è vol Maison des affaires culturels. Bagdad 1988.

BUCAILLE Maurice. La Torah, la Bible, le Coran et la science (Texte arabe). Le Bureau islamique. Beyrouth ,1990.

HAMIDULLAH Muhammad. Le Saint Coran .Amana Corporation. Maryland, USA .1989

Le NOBEL CORAN et la traduction en langue francaise de ses sens. Complexe Roi FAHAD pour l'impression du NOBEL CORAN. Al- Madina Al-Munawwarah. 1420 Héjrie. 
MUGHNIYAH Mohammed Jawad. Al Tafessir Al kashef. v1 (Texte arabe). Maison Al - Anwar Bayreuth. Liban. 2009.

REY Alain. Le Robert Micro. Le Robert. Montréal, Canada, 1998.

SABEK Jerwan. Dictionnaire Alkanze, (Texte arabe). Dar al Sabek, Beyrouth. 1972.

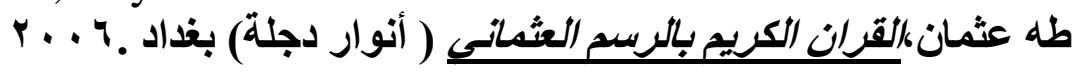


مصطلح (كلمة) في القرآن الكريم بين الترجمة والتفسير

المدرس: جمال عبد الحكيم عبد اله الجامعة المستتصرية/ كلية الآداب- قسم اللغة الفرنسية

ji200064@gmail.com

07702623722

(الملخص

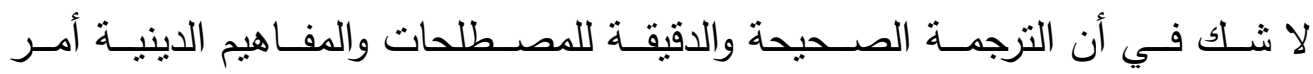

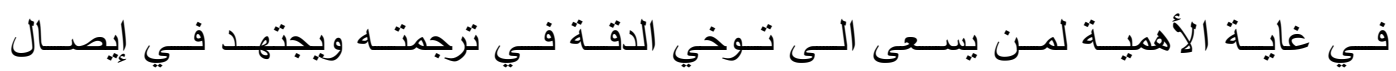

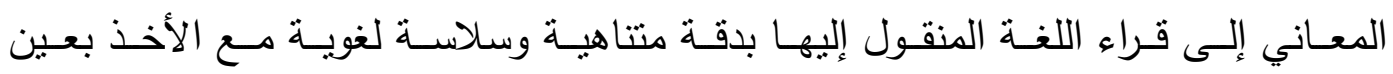

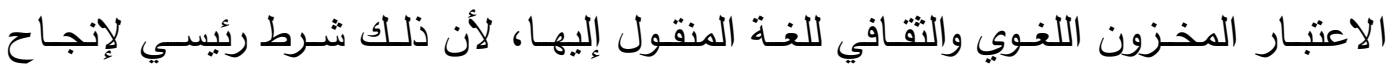

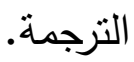

بسـى هـا البحـث إلـى إبـراز بلاغـة القـران مـن خـلال التطـرق إلى تعـدد معـاني

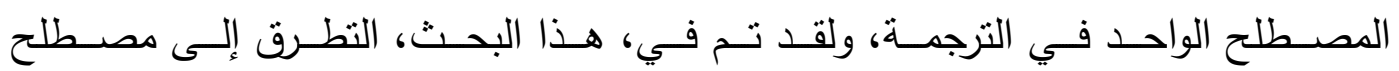
(كلمة) لورودها في r r اية في سور مختلفة من القران.

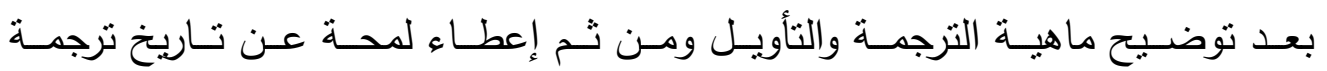

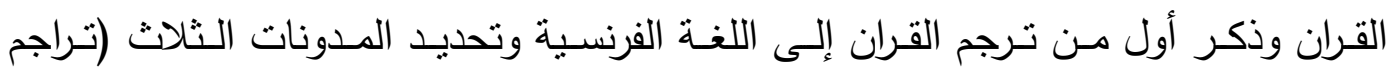

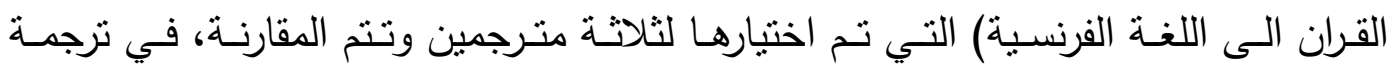

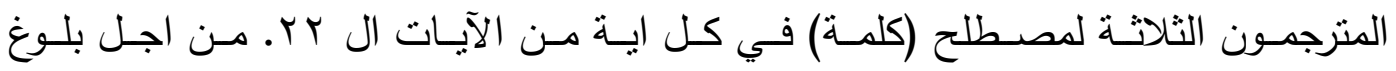
النتيجـة الدقيقة لهذه التـراجم تتم استتـارة نسختين مـن تفسيرات هذه الآيـات.مـن اجـل رصانة البحث، نم تمثيل النتائج بصيغة رسوم بيانية.

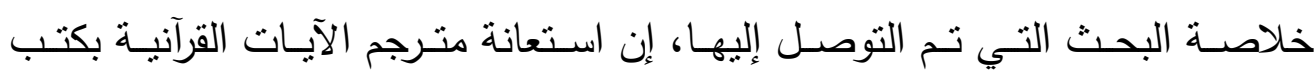

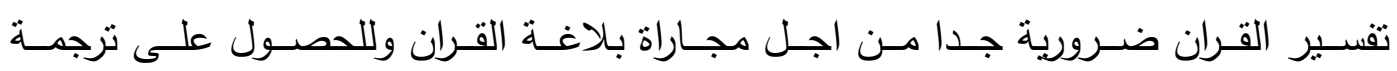

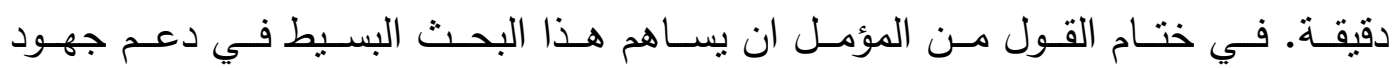
بحوث الترجمة بصورة عامة وترجمة القران بصورة خاصة.

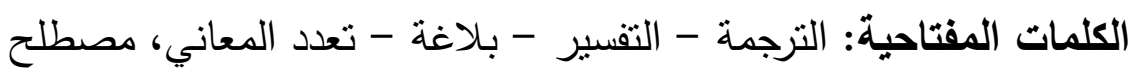

